\title{
Perceptions and Experiences of Latinx Parents with Language Barriers in a Pediatric Emergency Department: A Qualitative Study
}

Ronine L. Zamor ( $\sim$ Ronine.Zamor@emory.edu )

Cincinnati Children's Hospital Medical Center

Lisa M. Vaughn

Cincinnati Children's Hospital Medical Center

Erin McCann

Cincinnati Children's Hospital Medical Center

Luisanna Sanchez

Cincinnati Children's Hospital Medical Center

Erica M. Page

Cincinnati Children's Hospital Medical Center

E. Melinda Mahabee-Gittens

Cincinnati Children's Hospital Medical Center

\section{Research Article}

Keywords: Latinx, Pediatric Emergency Department, Language Barriers, Health Disparities

Posted Date: December 20th, 2021

DOI: https://doi.org/10.21203/rs.3.rs-1132498/v1

License: (1) This work is licensed under a Creative Commons Attribution 4.0 International License.

Read Full License 


\section{Abstract}

Background: Prior research has shown disparities exist among Latinx children who present to the pediatric emergency department (PED) for respiratory illnesses. Limited data exist regarding Latinx families' perspectives on healthcare for their children in PEDs within non-traditional destination areas. Their perspective can identify areas of improvement for reducing healthcare disparities among pediatric patients within this population. The purpose of this qualitative study was to explore the perceptions and experiences of Latinx families with language barriers in the PED.

Methods: Study staff screened the electronic health records of a purposive sample of patients. Families were considered eligible for the study if they: 1) presented to the PED with their 0-2 year-old child for a respiratory illness, 2) affirmed that their preferred language was Spanish at triage, and 3) requested a Spanish interpreter during registration. Semi-structured, one-on-one qualitative interviews were conducted via telephone, audiotaped, translated, and transcribed. All transcripts were reviewed using a thematic analysis.

Results: Interviews were conducted with 16 Latinx parents. Thematic analysis revealed four major themes: (1) Uncertainty_ - Families expressed uncertainty regarding how to care for a child with distressing symptoms, (2) Communication - Families favored in-person interpreters which enhanced communication and allowed families to feel more informed, (3) Resources - Families reported that the unfamiliarity with the US health system and lack of resources are additional burdens, and (4) The emergency department visits garnered confidence and reassurance for families.

Conclusions: The PED can be a key area of the health system that can help reduce disparities among the Latinx population. Further areas of intervention should focus on support beyond providing access to an interpreter, including identifying potential provider bias, improving information delivery, and enhancing education on community resources for families who face language barriers in the PED.

\section{Background}

In 2016, the Census Bureau estimated that the Hispanic population of the United States (U.S.) was 56.6 million, which constitutes $17 \%$ of the nation's total population. This proportion is expected to increase to $28 \%$ by 2060 (1). In the past, immigration of Latinx families to the U.S. has largely been concentrated in states with large urban areas, such as Texas, Florida, and New York (2). However, recent growth among the Latinx population has been observed in areas of the U.S. that were not common destination areas in the past, such as areas within the Midwest, which are defined as nontraditional destination areas (NDAs) (3). The rapid growth of the Latinx population has significant health implications because NDAs do not have the infrastructure and resources to support the changing immigration patterns $(4,5)$. Furthermore, literature describing Latinx healthcare experiences within NDAs remains limited.

Prior literature has shown significant healthcare disparities among Latinx children whose families face language barriers within the emergency department (ED) (6-9). For instance, there are differences in 
diagnostic testing and hospital charges in the pediatric emergency department (PED) among families with language barriers $(6,10,11)$. Some studies suggest that language barriers may be associated with poor health outcomes (7). Compared to Latinx patients in traditional destination areas, Latinx patients in NDAs have different healthcare barriers, experiences, and needs because healthcare systems in NDAs are not properly equipped to work with populations that have limited English proficiency $(3,4,12-14)$. Latinx patients in NDAs may face more challenges such as social isolation, discrimination, and lack of social support $(3,4,13,15)$. Given the rapid growth of Latinx population and the need for promoting greater equity in pediatric emergency care (16), exploring the experiences and perceptions of Latinx families surrounding their management in the PED can help target areas of improvement for reducing disparities within an NDA.

The purpose of this study is to explore the perceptions and experiences of non-English speaking Latinx families in the pediatric ED of a non-traditional destination area. The broader aim of this study is to target areas of improvement for reducing health care disparities for Latinx families.

\section{Methods}

\section{Study Setting}

The study was conducted at Cincinnati Children's Hospital Medical Center (CCHMC), one of the largest academic children's hospitals in the United States. The hospital treats children with rare and complex conditions from over 60 different countries (17). There are two different PEDs which in combination have approximately 92,000 visits per year. Upon arrival to the PED, families are asked their preferred language and whether they need an interpreter for the remainder of the visit. The preferred language is documented within the patient's chart. An interpreter can be used at any time or for the entire visit.

Physicians can use three different interpreter methods during a PED visit. The PED has an interpreter inperson nearly every day. If an in-person interpreter is not available, physicians have the option of using an interpreter via a video call. The last option is a telephone interpreter, though this method is the least common in the PED. At the time of this study, only one pediatric emergency medicine attending was considered a certified language interpreter in Spanish. All other physicians were required to use an interpreter if the patient and/or family did not speak English.

\section{Recruitment}

Study staff screened the electronic health records (EHR) of a purposive sample of patients to identify potential participants 7 days/week for an 8-month period. Parents or legal guardians were considered eligible for the study if they: 1) presented to the PED with their 0-2 year-old child for a viral upper or lower respiratory illness, 2) affirmed that their preferred language was Spanish at triage, and 3) requested a Spanish interpreter during registration. When screening was conducted in real-time while patients were in the PED, potentially eligible families were approached by study staff and a Spanish interpreter to explain the study, review informed consent, and schedule a telephone interview once informed verbal consent 
was obtained. The researchers recruited families in the PED with respiratory illnesses given the known disparities among pediatric patients with respiratory complaints within this population (6).

Study staff also conducted weekly screening of the EHRs to identify potentially missed eligible families who had already been discharged. If the family met the inclusion criteria, study staff called the family to inform them of the study, solicit participation, and obtain informed verbal consent. The patient's age, medical history, chief complaint, and use and type of interpreter involved with the family was reviewed and confirmed prior to proceeding with the interview. All participants were given a \$20 gift card in exchange for their participation in the study.

\section{Data Collection}

This study drew from the phenomenology framework developed by Max Van Manen and Martin Heidegger, who described a hermeneutic approach to phenomenology that stresses how human experiences and "realities" are influenced by the world in which they live. This framework provided a way to understand each family's meaning that they construct from their experiences in the PED $(18,19)$. Three authors conducted 16 semi-structured, one-on-one qualitative interviews from May 2019 through January 2020. Two of the three interviewers were fluent in Spanish and did not use an interpreter for the interviews. The interviewers used an interview guide (Table 1) that allowed them the flexibility to deviate from the guide and ask probing questions based on participants' responses.

Table 1

Interview Guide

\section{Where are you from? How long have you lived in the United States?}

2. What resources do you use in your community when your child is sick?

3. If you remember, what brought you and your child to the emergency department?

4. What concerned you the most about your child's symptoms when you visited the emergency department?

5. Do you remember if you took your child to his/her primary doctor before going the emergency department that day? Tell me about that.

6. Tell me what the several days before you took your child to the emergency department were for you.

7. Describe to me how your visit went. Ex: Testing, treatments, expectations

\section{Was there anything done that day that you think helped your child feel better?}

9. Describe your understanding of why things were or were not done.

10. How did your doctor communicate with you when deciding what should or should not be done?

11. Describe how you communicated what concerned you regarding your child during your visit.

12. What things did you not tell your doctor that you wish you had told them?

13. How did you feel having an interpreter there helped or did not help with your visit that day?

14. How do you think communication about what was done during your visit could have been better? 
All families who gave informed verbal consent within the PED had their interview completed via phone within 30 days of the index PED visit. All interviews were audiotaped then translated and transcribed. Interviews were conducted until thematic saturation was reached, which occurred when the interviewers did not elicit any new information or themes (20).

\section{Data Analysis}

The research team utilized a thematic analysis approach to systematically identify meaningful subthemes and themes that represented major topics discussed in the interviews $(21,22)$. Thematic analysis involved an iterative process to further clarify the themes that could be presented in a cohesive narrative. First, the research team independently reviewed all interview transcripts and developed codes, which were key words that represented specific phrases, patterns and common features found in the transcripts. A codebook was developed to help guide the data analysis. All members of the research team collectively reviewed and coded all transcripts. The research team met regularly to review the transcripts and discuss emerging codes until code agreement and saturation were reached. Each code was collated into unifying theme along with subcategories within each theme. The software program Dedoose was used to highlight specific themes or common phrases that might have been missed when reviewing the transcripts as a group (23). Code frequencies within Dedoose were also used to identify patterns in families' responses (23).

\section{Results}

\section{Characteristics of Study Participants}

Interviews were conducted with 16 Latinx parents (15 mothers, 1 father) who were seen in the PED with a child presenting with respiratory symptoms. The length of interviews ranged from 11 minutes to 26 minutes (median, 20 minutes). Most pediatric patients were male (66\%). Table 2 shows the proportions of patient triage levels, chief complaints, disposition, and final diagnoses. 
Table 2

Characteristics of 16 Participating Families

\begin{tabular}{|c|c|}
\hline Characteristic & No. (\%) \\
\hline Patient Gender Male & $9(56 \%)$ \\
\hline \multicolumn{2}{|l|}{ Family Country of Origin } \\
\hline Mexico & $4(25 \%)$ \\
\hline Guatemala & $5(31 \%)$ \\
\hline Puerto Rico & $1(6 \%)$ \\
\hline No answer & $6(38 \%)$ \\
\hline \multicolumn{2}{|l|}{ Chief Complaint } \\
\hline Fever and/or difficulty breathing & $7(44 \%)$ \\
\hline Fever and/or cough & $6(38 \%)$ \\
\hline Nasal congestion & $1(6 \%)$ \\
\hline Fever & $2(12 \%)$ \\
\hline \multicolumn{2}{|l|}{ Triage Acuity } \\
\hline 2 (Most severe) & $3(19 \%)$ \\
\hline 3 & $7(44 \%)$ \\
\hline 4 (Least severe) & $6(38 \%)$ \\
\hline \multicolumn{2}{|l|}{ Final ED in Diagnosis } \\
\hline Bronchiolitis & $9(56 \%)$ \\
\hline Viral illness/Cold/Fever & $4(25 \%)$ \\
\hline Croup & $1(6 \%)$ \\
\hline Pneumonia & $1(6 \%)$ \\
\hline Bilateral Otitis Media & $1(6 \%)$ \\
\hline \multicolumn{2}{|l|}{ Method of Interpreter Used } \\
\hline In-Person & $6(38 \%)$ \\
\hline Video & $5(31 \%)$ \\
\hline Phone & $5(31 \%)$ \\
\hline Admitted & $6(37 \%)$ \\
\hline
\end{tabular}


Four major themes emerged from the thematic analysis: (1) Uncertainty - fear and uncertainty regarding how to care for a child with distressing symptoms, (2) Communication - the presence of in-person interpreters enhanced communication, allowing parents to feel more informed, (3) Resources unfamiliarity with the U.S. healthcare system and lack of resources are additional burdens and (4) Closure - despite limited understanding, "successful" visits garnered confidence and provided parents with needed reassurance. Table 3 lists illustrative quotations representative of major themes and subthemes. 
Table 3

Themes and Subthemes with Illustrative Quotations

\section{Themes and Subthemes, With Illustrative Quotations}

\section{Theme or Subtheme Illustrative Quotation}

Uncertainty

Seriousness

Fear

Biggest Concern

from parents

Confidence in one's ability
"But that was just the fever but the thing I was worried the most about was his chest"

"Oh, I was worried about my little girl dying of the disease, so I wanted to get her to the emergency room quickly"

"He had constant fever and trouble breathing"

"The pediatrician's office is nearby like eight minutes away from here. I always have Tylenol at home. I have the suction pump. I also have the machine"

\section{Communication}

Communication during visit (intake, history)

"They examined him and they told me that he was very sick so they gave him oxygen and they told me that the doctor would come to examine him and tell me if he was going to be hospitalized or not. Half an hour later the doctor came and he told us that he needed to stay"

Informed

"Yes, they explained how everything was going to be done and I was ok with that"

Method of

Communication

"Yes, having the in-person interpreter over there was better"

Understanding of

"Well they were treating his nose, for the baby to breathe well"

diagnosis/Treatment

plan

Resources

Community

resources

Familiarity with

healthcare system

"Yeah, he has a social worker"

"So, it's very different. I have been living here for like two years but the way they treat you here is very different compared to the way they treat you over there when you come to the emergency room with a child. In Puerto Rico, they would run a CBC test, and they would run lab tests, and ultrasound. And that's not how it is here"

Navigating the

System

Access

Family member
"No, not for him, no obstacles. For me yes; I haven't found a way to get into health insurance"

"Yes, she's going to a clinic, well I take her to a clinic called Price Hill. It's' about 5 minutes from here"

"My sister in law told the girl to find a girl who spoke English so I could explain things to her" 


\section{Themes and Subthemes, With Illustrative Quotations}

Closure

Reassurance

Physician's authority
"Thanks to God, everything was going good"

"I did expect something else, but I was fine with it, they're the doctors, of course"

"They did everything my baby needed"

"I felt confident in my doctors and they took away that doubt and that anguish"

\section{Uncertainty regarding how to care for a child with distressing symptoms:}

I was worried about my little girl dying of this disease." "Maybe if it was another case, my baby might have died, because we don't know how to care of the baby in the house.

Participants reported that their children were experiencing various symptoms that were disconcerting. Parents were frightened by the symptoms and were unsure of their ability to care for their child at home. Some participants reported that they did not provide any treatment at home because they were inexperienced as a parent and thus not sure of the appropriate course of action. Others believed that if they provided interventions, they might make the situation worse. Furthermore, parents were fearful that if they did seek medical treatment, their limited English proficiency would affect their ability to effectively communicate with medical professionals. Nevertheless, their child's distressing symptoms prompted parents to seek help anyway.

\section{In-person interpreters enhanced communication, allowing parents to feel more informed: "They} explained how everything was going to be done and I was okay with that." "It did help me a lot because that way I was able to communicate through this person. I was able to explain everything. I was able to explain and say everything - everything that was going on with my son. And everything the doctor and the nurse wanted to tell me I was able to understand all of it through this person"

Participants reported that providers attempted to explain their child's diagnosis and treatment plan; however, limited understanding of medical terminology was a barrier. Despite this issue, in-person interpreters lessened parents' confusion and improved communication by translating parental concerns and describing procedures and tests discussed by the provider. The frequency of reported understanding was higher with in-person and video interpreters. To facilitate understanding, one participant reported that their provider showed a video depicting symptoms that would require medical attention. These measures (i.e., videos, pictures, infographics) made most parents feel informed of the plan of care and few reported feeling ignored and uninformed.

3. Unfamiliarity with the U.S. healthcare system and lack of resources are additional burdens: "The way you are treated here is very different." "I mean, for them to do something it has to be a life or death situation." 
Parental expectations for care in the U.S. were affected by care they had previously received in their home country. Some parents believed that in order to receive the standard of care they are accustomed to in the PED, their child must be severely ill. Participants also reported having trouble making appointments with primary care pediatricians and limited accessibility to clinics that provide interpreters. Some parents listed social workers and family members accustomed to the system as sources of advice and assistance in coordinating care and accessing treatment. However, many participants reported social isolation and few community resources.

4. "Successful" visits garnered confidence and provided needed reassurance: "I felt confident in my doctors and they took away that doubt and that anguish." "They did everything my baby needed." Although parents did not completely understand their provider, many were satisfied with the visit. Most participants believed their providers adequately addressed their concerns and provided adequate care to their children. However, a few parents felt the appointment was rushed and their concerns were not taken seriously due to the mild nature of their child's illness. Overall, participants trusted their providers and were calmed by their provider's assurance about their child's prognosis.

\section{Discussion}

This study explored parental perspectives regarding the treatment of Latinx children when presenting to a PED with respiratory symptoms. Qualitative studies such as this study can help identify specific factors from the parents' point of view that could be improved to reduce disparities related to language barriers. This is extremely important for PED providers because they are treating patients at one point in time, without much, if any, foreknowledge of what led the families to their visit. Since most information is obtained from the triage process, history and chart review, there is no simple way to explore how families experienced the events that occurred during an ED visit. Prior research studies have focused on the discharge process as a means for improving patient outcomes. For example, interventions such as formally structuring the discharge process have been shown to improve disparities in post-discharge outcomes by reducing medication errors and return visits to the ED (24). However, interventions can also be targeted towards disparities identified by parents' experiences prior to discharge. In this study, the major themes that emerged included the fear of progression of illness, uncertainty about the child's clinical status, the importance of using in-person interpreters, unfamiliarity with the healthcare system and the shift in perspective from lack of confidence to confidence in their ability to care for their child after the healthcare provider's diagnosis and management in the PED.

This study reinforced the importance of having in-person and/or video interpreters, which is a relatively simple but vital aspect of a parents' experience when presenting to a PED for any health concern while facing a language barrier. Families felt more informed and reassured when a video or a combination of an in-person and video interpreter were used during the PED visit. It is known that the use of professional interpreters is associated with overall improvement in care for patients with limited English proficiency, with in-person or video interpretation leading to higher patient satisfaction compared to telephone interpretation $(25,26)$. Prior research examining the Latinx population has shown that a lack of staff 
members of the same ethnic or language group is associated with barriers to health care $(13,27)$. Although several prior research studies show that families may still have a degree of mistrust regardless of having access to an interpreter $(28,29)$, families in this study reaffirmed that they remained informed and reassured when they were given the opportunity to interact with an interpreter in-person or through a video.

Another area for targeted interventions identified in this study is related to navigating the healthcare system. Disparities in PED management may be due to a lack of access to primary care for Spanishspeakers. Families often reported trying to visit primary care clinics first, but they were unable to get a visit with a Spanish-speaking provider or they did not know what to do if it was after regular business hours. These experiences support the literature that suggests increased PED utilization for routine sick visits among Latinx children, resulting in fewer interventions in the PED due to the higher frequency of low acuity visits $(9,28)$. Navigating the healthcare system can be more difficult among Latinx families within an NDA. Lower acculturation among ethnic groups has been shown to be associated with higher numbers of PED visits (27). The increased difficulty for Latinx families within an NDA can lead to even higher numbers of PED visit and may influence their care in the emergency setting. Patients among families who had difficulty with navigating the healthcare system had lower triage levels (higher acuity). The PED may be another setting in which families can receive education on the availability and location of community resources. Families could receive education about the healthcare system-how to seek care outside of clinic hours and what to expect from an urgent care or emergency department visit. These families may come from countries with limited community supports and may benefit from a clear explanation of community programs that are available. PEDs can coordinate with local pediatric offices to facilitate these connections by providing information in Spanish about available resources in the community.

Another key issue highlighted in this study was the importance of educating the family about their child's diagnosis and management in the PED. Families were often very grateful for the treatment they received even if the intervention was minimal such as nasal suctioning or providing an antipyretic. However, it was not clear if parents always understood their child's diagnosis or return precautions because of language barriers or health literacy levels. One family expressed appreciation for a short video that was shared with them before discharge that demonstrated an infant in respiratory distress as this ensured they understood appropriate return precautions. Short, educational videos focused on common PED diagnoses could be a useful tool that is relatively easy to create in a patient's native language. Furthermore, healthcare providers can show the video and then answer any follow-up questions without having to spend a lengthy amount of time providing anticipatory guidance to families. These videos could be shared online as well, so families have access to educational materials even before presenting to an emergency department. Increasing educational tools can not only increase understanding but can provide tremendous support especially for families who face increased social isolation and lack of community engagement within an NDA. 
This study demonstrates that the PED is a key area of the healthcare system that can help reduce health disparities among the Latinx population. Most parents reported lack of knowledge of community resources, and presented with lower acuity visits to the ED. Although the need for extensive testing and interventions was low in this participant cohort, parents were very thankful and reassured for the care they received in the PED. This finding is interesting in the light of prior quantitative findings showing that Latinx patients facing language barriers receive increased PED charges and increased testing compared to patients without language barriers. Although patient level factors may lead to differences in acuity levels among patients with language barriers, disparities in care during the PED visit may be more influenced by factors unrelated to the patients themselves, such as provider bias or access to care (29). Our results indicate that possible ways to reduce disparities among patients with language barriers include addressing potential areas of provider bias while increasing support and coordination with community resources for this vulnerable population.

There were several limitations that should be noted. Two of the three interviewers were not from the Latinx community, which could have impacted participants' willingness to be open and honest. Although data reached thematic saturation (20), the majority of the interviewed families were from the same country (e.g., Guatemala). Thus, findings should be interpreted with caution and may not apply to other Latinx populations. Lastly, although intentional in qualitative research, the study was conducted at a single site with non-probability sampling which limits the applicability of findings to other clinical settings.

\section{Conclusions}

This qualitative study explored the perceptions and experiences of Latinx families after a PED visit within an NDA. There were four main themes among families of young children presenting with respiratory illnesses. All families were fearful of their child's illness, but they appreciated being informed via an interpreter and felt reassured by the end of the PED visit. However, families did have difficulty navigating the healthcare system. The PED can be a key setting within the healthcare system that can help reduce disparities among the Latinx population within an NDA. Further areas of intervention should focus on support beyond providing access to an interpreter, such as identifying potential provider bias, improving information delivery, and enhancing education on community resources for families who face language barriers in the PED.

\section{Abbreviations}

Pediatric Emergency Department - PED

Emergency Department - ED

Nontraditional Destination Area - NDA

Cincinnati Children's Hospital Medical Center - CCHMC

Page $12 / 16$ 


\section{Declarations}

\section{Ethics approval and informed verbal consent to participate}

The study was approved by the Institutional Review Board at CCHMC and the research team followed ethical guidelines corresponding to research with human subjects. Informed verbal consent was obtained from all participants.

\section{Consent for publication}

Not Applicable

\section{Availability of data and materials}

Not Applicable

\section{Competing interests}

The authors declare that they have no competing interests.

\section{Funding}

This work was funded by the Eunice Kennedy Shriver National Institute of Child Health and Human Development: NIH Grant Number R01HD083354. This funding source had no involvement in the study design, data collection, analysis, or interpretation of data.

\section{Authors' contributions}

RZ, LV and EMM conceived the study and the overall study design. LV and EMM supervised the conduct of the study. RZ, EM, and LS recruited participants and conducted the interviews. RZ, EM, LS and EP analyzed the data. All authors reviewed the data and results. RZ, EM, LS, and EP drafted the manuscript and all authors contributed substantially to its revision. All authors approved the final manuscript as submitted and agree to be accountable for all aspects of the work.

\section{Acknowledgements}

Not Applicable

\section{References}

1. Cortez R. Hispanic Heritage Month. Not Am Math Soc. 2018;65(08):1. 
2. Passel JS, Cohn D, Lopez MH. Hispanics Account for More than Half of Nation's Growth in Past Decade I Pew Research Center. Pew Hisp Cent [Internet]. 2011; Available from: http://www.pewhispanic.org/2011/03/24/hispanics-account-for-more-than-half-of-nations-growth-inpast-decade/

3. Jacquez F, Vaughn L, Zhen-Duan J, Graham C. Health care use and barriers to care among Latino immigrants in a new migration area. J Health Care Poor Underserved. 2016;27(4):1761-78.

4. Jacquez F, Vaughn LM, Pelley T, Topmiller M. Healthcare Experiences of Latinos in a Nontraditional Destination Area. J Community Pract. 2015;23(1):76-101.

5. DeJonckheere MJ, Vaughn LM, Jacquez F. Latino Immigrant Youth Living in a Nontraditional Migration City: A Social-Ecological Examination of the Complexities of Stress and Resilience. Urban Educ. 2017;

6. Zamor R, Byczkowski T, Zhang Y, Vaughn L, Mahabee-Gittens EM. Language Barriers and the Management of Bronchiolitis in a Pediatric Emergency Department. Acad Pediatr. 2020;20(3):356-63.

7. Levas MN, Dayan PS, Mittal MK, Stevenson MD, Bachur RG, Dudley NC, et al. Effect of hispanic ethnicity and language barriers on appendiceal perforation rates and imaging in children. $\mathrm{J}$ Pediatr. 2014;164(6).

8. Valet RS, Gebretsadik T, Carroll KN, Minton PA, Woodward KB, Liu Z, et al. Increased healthcare resource utilization for acute respiratory illness among Latino infants. J Pediatr. 2013;163(4):1186-91.

9. Lassetter $\mathrm{JH}$, Baldwin $\mathrm{JH}$. Health care barriers for latino children and provision of culturally competent care. J Pediatr Nurs. 2004;19(3):184-92.

10. Hampers LC, Cha S, Gutglass DJ, Binns HJ, Krug SE. Language barriers and resource utilization in a pediatric emergency department. Pediatrics. 1999;103(6 I):1253-6.

11. Payne NR, Puumala SE. Racial disparities in ordering laboratory and radiology tests for pediatric patients in the emergency department. Pediatr Emerg Care. 2013;29(5):598-606.

12. Jacquez F, Vaughn LM, Suarez-Cano G. Implementation of a Stress Intervention with Latino Immigrants in a Non-traditional Migration City. J Immigr Minor Heal. 2019;21(2):372-82.

13. Topmiller M, Zhen-Duan J, Jacquez FJ, Vaughn LM. Place Matters in Non-Traditional Migration Areas: Exploring Barriers to Healthcare for Latino Immigrants by Region, Neighborhood, and Community Health Center. J Racial Ethn Heal Disparities. 2017;4(6):1214-23.

14. Zhen-Duan J, Jacquez F, Vaughn L. Demographic characteristics associated with barriers to health care among Mexican and Guatemalan immigrants in a nontraditional destination area. Fam Community Heal. 2017;40(2):101-11. 
15. Biolsi R. Our Diverse Society: Race and Ethnicity-Implications for 21 st Century American Society, by David W. Engstrom and Lissette M. Piedra, (eds.) . Adm Soc Work [Internet]. 2009 Jan 5;33(1):129-31. Available from: https://doi.org/10.1080/03643100802508700

16. McDermott KW, Stocks C, Freeman WJ. Overview of Pediatric Emergency Department Visits, 2015 : Statistical Brief \#242 [Internet]. Healthcare Cost and Utilization Project (HCUP) Statistical Briefs. Rockville, MD; 2006 [cited 2020 Aug 31]. Available from: http://www.ncbi.nlm.nih.gov/pubmed/30277692

17. Cincinnati Children's Hospitals. Cincinnati Children's: International Patients and Families [Internet]. 2016 [cited 2018 Oct 8]. Available from: https://www.cincinnatichildrens.org/patients/visit/international

18. Moustakas C. Phenomenological research methods. Phenomenological research methods. 2011.

19. Crotty M. The foundations of social research: Meaning and perspective in the research process. Sage. 1998.

20. Bowen GA. Naturalistic inquiry and the saturation concept: A research note. Qual Res. 2008;8(1):13752.

21. Hsieh HF, Shannon SE. Three approaches to qualitative content analysis. Qual Health Res. 2005;15(9):1277-88.

22. Braun V, Clarke V. Using thematic analysis in psychology. Qual Res Psychol. 2006;3(2):77-101.

23. Dedoose version 8.0.35. Dedoose. dedoose.com. 2018.

24. Samuels-Kalow M, Hardy E, Rhodes K, Mollen C. “Like a dialogue”: Teach-back in the emergency department. Patient Educ Couns. 2016;99(4):549-54.

25. Karliner LS, Jacobs EA, Chen AH, Mutha S. Do professional interpreters improve clinical care for patients with limited english proficiency? A systematic review of the literature. Health Serv Res. 2007;42(2):727-54.

26. Casey Lion K, Brown JC, Ebel BE, Klein EJ, Strelitz B, Gutman CK, et al. Effect of telephone vs video interpretation on parent comprehension, communication, and utilization in the pediatric emergency department a randomized clinical trial. JAMA Pediatr. 2015;169(12):1117-25.

27. Vaughn LM, Jacquez F. Characteristics of newly immigrated, Spanish-speaking Latinos who use the pediatric emergency department: Preliminary findings in a secondary migration city. Pediatr Emerg Care. 2012;28(4):345-50.

28. Fields A, Abraham M, Gaughan J, Haines C, Hoehn KS. Language matters: Race, trust, and outcomes in the pediatric emergency department. Pediatr Emerg Care. 2016;32(4):222-6. 
29. Mayo RM, Sherrill WW, Sundareswaran P, Crew L. Attitudes and perceptions of hispanic patients and health care providers in the treatment of hispanic patients: A review of the literature. Vol. 5, Hispanic Health Care International. 2007. p. 64-72. 